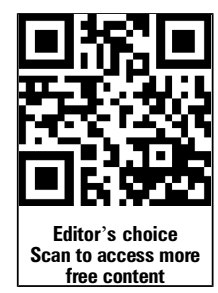

Handling editor Tore K Kvien

- Additional material is published online only. To view please visit the journal online (http://dx.doi.org/10.1136/ annrheumdis-2014-206047)

'Department of Rheumatology, Leiden University Medical

Center, Leiden,

The Netherlands

${ }^{2}$ Department of Medical Statistics and Bioinformatics, Leiden University Medical

Center, Leiden,

The Netherlands

${ }^{3}$ Department of Rheumatology, Nîmes University Hospital, EA

2415, Montpellier I University, Nîmes, France

${ }^{4}$ Université Pierre et Marie Curie Curie-Paris 6, GRC08, Institut Pierre Louis d'Epidémiologie et Santé Publiqu, Paris, France

\section{Correspondence to} Jessica A.B. van Nies, Department of Rheumatology, Leiden University Medical Center, P.O. Box 9600, Leiden 2300 RC, The Netherlands; j.a.b.van_nies@lumc.nl

Received 6 June 2014 Revised 6 December 2014 Accepted 8 December 2014 Published Online First

5 January 2015

CrossMark

\section{SLinked}

- http://dx.doi.org/10.1136/ annrheumdis-2014-206993

To cite: van Nies JAB, Tsonaka R, Gaujoux-Viala C, et al. Ann Rheum Dis 2015;74:806-812.

\title{
Evaluating relationships between symptom duration and persistence of rheumatoid arthritis: does a window of opportunity exist? Results on the Leiden Early Arthritis Clinic and ESPOIR cohorts
}

\author{
J A B van Nies, ${ }^{1}$ R Tsonaka, ${ }^{2}$ C Gaujoux-Viala, ${ }^{3}$ B Fautrel, ${ }^{4}$ \\ A $\mathrm{H} M$ van der Helm-van Mil ${ }^{1}$
}

\section{ABSTRACT}

Background A prolonged symptom or disease duration at treatment initiation is associated with unfavourable outcomes in rheumatoid arthritis (RA). It is unknown whether this relation is linear, referring to a common 'the-earlier-the-better principle', or whether a transient time frame in which the disease is more susceptible to treatment exists, referring to a 'window of opportunity'. To elucidate this, we evaluated the shape of the associations of symptom duration with persistence of RA. Methods Patients with 1987 RA treated with disease modifying antirheumatic drugs (DMARDs) in the Leiden Early Arthritis Clinic (EAC, $n=738$ ) and Evaluation et Suivi de POlyarthrites Indifférenciées Récentes (ESPOIR) $(n=533)$ were studied. Cox proportional hazards regression models using natural cubic splines were performed; the log-HR on DMARD-free sustained remission (the opposite of RA persistence) during 5-year follow-up was plotted against symptom duration. Discrimination was measured using time-dependent receiver operator characteristic curves. Subanalyses were performed stratified for the DMARDs used (methotrexate or other conventional DMARDs) and for anticitrullinated peptide antibody (ACPA).

Results $11.5 \%$ (85/738) and 5.4\% (29/533) of EAC and ESPOIR RA patients achieved DMARD-free sustained remission. In both cohorts and all analyses, the curves depicting the log-HRs on remission in relation to symptom duration were not linear. The symptom duration with optimal discriminative ability was 14.9 weeks $(95 \% \mathrm{Cl} 12.3$ to 16.0 ; area under the curve (AUC) 0.61$)$ in the EAC and 19.1 weeks (95\% CI 12.3 to 28.0; AUC 0.59) in ESPOIR. For ACPA-positive RA, this was 11.4 weeks (95\% Cl 7.7 to 79.0 ; AUC 0.56) and for ACPA-negative RA 15.0 weeks $(95 \% \mathrm{Cl} 9.7$ to 48.7; AUC 0.56).

Conclusions The association between symptom duration and RA persistence is not linear, suggesting the presence of a confined period in which RA is more susceptible to treatment.

\section{INTRODUCTION}

Many studies within rheumatoid arthritis (RA) have shown that a prolonged disease duration or symptom duration at treatment initiation is unfavourable. It is associated with more severe radiographic joint destruction, a higher need of orthopaedic surgery, a higher mortality rate and a lower chance to achieve disease modifying antirheumatic drug (DMARD)-free sustained remission. ${ }^{1-5}$ The shape of the association between the risk on an unfavourable disease course and the symptom duration at treatment start, however, is not known.

Two profiles may possibly explain the mentioned observations. First, a transient window during which outcomes can be more effectively modulated by therapy may be present. This refers to the 'window of opportunity' hypothesis and implies that whenever certain master-switches (crucial processes) have turned on or off the responsiveness to treatment is reduced. A small observational study aiming to evaluate the optimal time point of DMARD introduction showed that patients with a median disease duration of 3 months achieved lower disease activity score (DAS) scores and less radiographic damage than patients with a median disease duration of 12 months at first DMARD initiation. ${ }^{6}$ Two larger cohort studies observed that patients with symptom durations $\leq 12$ weeks developed less joint destruction ${ }^{2}{ }^{7}$ and achieved DMARD-free sustained remission more often $^{2}$ than patients with symptom durations $>12$ weeks. Although these observations may support the presence of a temporary window of opportunity, it has never been shown that after a certain symptom duration the effect of initiated treatment starts to diminish.

The alternative explanation of the association between the time till intervention and the outcome of RA relates to the 'the-earlier-the-better' principle that is universally valid in the context of the initiation of treatment for many diseases. ${ }^{8}$ With regard to RA, it can be presumed that the total inflammatory load at the time of treatment onset is related to the disease outcome. Since the total inflammatory load is the product of the severity and the duration of inflammation, a linear association between the symptom duration and the outcome of RA can be hypothesised. Then the effect of early intervention is not confined to a very early window. In 1995, a randomised clinical trial in recent onset RA patients was published; ${ }^{9}$ patients started treatment with auranofin directly or after a delay of 8 months. The early treatment group had better outcomes and this effect was maintained after 5-year follow-up. A similar trial was performed using hydroxychloroquine and similar findings were obtained. ${ }^{10}$ The timing of intervention in most patients in both arms of both studies was considerably later than $<3$ months of symptom onset and still early 
treatment initiation was beneficial. Therefore, the advantageous effect of early treatment might not be restricted to a very early and temporary time frame. Furthermore, the difference in outcome observed in cohort studies comparing patients with $<$ or $\geq 3$ months symptoms ${ }^{267}$ can also be found in case symptom duration is linearly associated with the outcome. Moreover, a recent study evaluating symptom duration on a continuous scale using regular cox regression, thus assuming that every increase in symptom duration is equally deleterious and hence assuming linearity, also observed significant associations for symptom duration. ${ }^{1}$

Altogether, these studies do not allow differentiating between a linear effect and a window of opportunity. This study, therefore, set out to elucidate the shape of the relationship between the symptom duration at treatment initiation and the risk on a persistent course of RA. Persistence was defined as the absence of DMARD-free sustained remission; of possible disease outcomes, this outcome is the closest available proxy of cure of RA. We studied RA patients treated with conventional DMARDs that were included in two early arthritis cohorts. Symptom duration was kept continuous. The cox regression model applied did not assume a particular form of the relationship and the model that fitted the data best was presented.

\section{METHODS}

\section{Patients}

RA patients were classified according to the 1987 American College of Rheumatology (ACR) criteria and derived from the Leiden Early Arthritis Clinic (EAC) and the French Evaluation et Suivi de POlyarthrites Indifférenciées Récentes (ESPOIR) cohort.

The EAC is an inception cohort that started in 1993 and has been described previously. ${ }^{11}$ Inclusion took place when arthritis was confirmed by physical examination and symptom duration was $<2$ years. At study entry, patients used no DMARDs or glucocorticoids. For the current research question, RA patients who were promptly treated with DMARD therapy after inclusion were selected. Between 1996 and 2011, this concerned 802 RA patients; 738 of these had information on dates of symptom onset and were studied. The 63 patients without this information and the 738 studied patients did not differ in baseline characteristics (data not shown). Information on the initiated DMARDs is presented in table 1 . None of the patients included in the years 1993-1995 were evaluated, as initial therapy concerned nonsteroidal anti-inflammatory drugs (NSAIDs) but not DMARDs.

ESPOIR is a longitudinal cohort including patients with RA or a suspicion to develop RA from 14 rheumatology centres in France. ${ }^{12}$ Patients had to be aged $18-70$ years and have $\geq 2$ swollen joints for $>6$ weeks and $<6$ months. Out of the 813 RA patients included between 2002 and 2005, 533 were treated with DMARDs and studied here.

In both cohorts, patients filled in questionnaires at baseline, joint counts were performed and laboratory evaluations done. The yearly follow-up visits included clinical, laboratory and radiographic evaluations.

Symptom onset in EAC and ESPOIR was delineated identically and defined as the first musculoskeletal symptom (either being pain or swelling) relevant to the current presentation. ${ }^{13}$ The symptom duration was defined as the duration between this patient reported symptom onset and inclusion and was determined by subtraction of dates, expressed in weeks.

\section{Outcome}

The main outcome was DMARD-free sustained remission; this is the opposite of disease persistence and was defined as the sustained absence of arthritis (absence of swelling by physical
Table 1 Characteristics of the RA patients studied

\begin{tabular}{|c|c|c|}
\hline & EAC $n=738$ & ESPOIR $n=533$ \\
\hline Age, years, mean $\pm S D$ & $57.2 \pm 15.7$ & $48.8 \pm 11.9$ \\
\hline Gender, n (\%) & $484(65.6)$ & $404(75.8)$ \\
\hline $\begin{array}{l}\text { Symptom duration at first visit, } \\
\text { weeks, median (IQR) }\end{array}$ & $18.7(9.3-35.3)$ & $21.3(13.4-33.5)$ \\
\hline SJC, median (IQR) & $8.0(4.0-13.5)$ & $7.0(4.0-12.0)$ \\
\hline RF-positive, n (\%) & $430(58.7)$ & $309(58.0)$ \\
\hline ACPA-positive, n (\%) & $370(52.4)$ & $268(50.3)$ \\
\hline ESR, mm/h, median (IQR) & $31.0(17.0-50.0)$ & $24.0(12.0-44.5)$ \\
\hline \multicolumn{3}{|l|}{ Initiated DMARD, n (\%) } \\
\hline Methotrexate & $563(76.3)$ & $357(67.0)$ \\
\hline Sulfasalazine & $100(13.6)$ & $62(11.6)$ \\
\hline Hydroxychloroquine & $63(8.5)$ & $64(12.0)$ \\
\hline Leflunomide & - & $30(5.6)$ \\
\hline Other DMARDs* & $12(1.6)$ & $20(3.8)$ \\
\hline \multicolumn{3}{|c|}{$\begin{array}{l}\text { Missing data were as follows: in the EAC, SJC n=17, RF n=5, ACPA n=37, ESR n=6; } \\
\text { in ESPOIR, ESR } n=5 \text {. } \\
\text { *Including glucocorticoids. } \\
\text { ACPA, anticitrullinated peptide antibody; DMARD, disease modifying antirheumatic } \\
\text { drug; EAC, Early Arthritis Clinic; ESPOIR, Evaluation et Suivi de POlyarthrites } \\
\text { Indifférenciées Récentes; ESR, erythrocyte sedimentation rate; RA, rheumatoid } \\
\text { arthritis; RF, rheumatoid factor; SJC, swollen joint count. }\end{array}$} \\
\hline
\end{tabular}

examination) after discontinuation of DMARD therapy, including biologics and glucocorticoids (systemic and intra-articular), for the entire period of follow-up (at least 1 year, up to 5 years). In the EAC, all medical files were explored until 5 April 2012. In ESPOIR, all structured visits in the database were reviewed.

\section{Subanalyses}

Because it is not known whether treatment with different DMARDs differently affects the associations between symptom duration and the log-hazard on DMARD-free sustained remission, analyses were stratified for the use of methotrexate (the current first-line therapy) and other conventional DMARDs. Further, since the biological pathway of development of anticitrullinated peptide antibody (ACPA)-positive and ACPA-negative RA is different, and ACPA-positive RA patients achieve DMARD-free sustained remission less often than ACPA-negative patients, it is possible that the associations between symptom duration and the log-hazard on DMARD-free sustained remission differ for ACPA-positive and ACPA-negative RA. Analyses were therefore also stratified for ACPA. Finally, we assessed the shape of the association of symptom duration with other outcomes. Although the outcome DMARD-free sustained remission (and its counterpart RA persistence) most closely fits with the window-of-opportunity hypothesis, DMARD-free sustained remission is achieved infrequently and for clinical daily practice other outcomes are relevant as well. The first alternative outcome was radiological joint destruction. The time between baseline and achieving a certain progression in joint destruction was assessed; two cut-offs were chosen rather arbitrarily and applied in the EAC in 485 RA patients who were included between 1996 and 2006 with scored radiographs (by one experienced reader with an intraclass observer correlation coefficients of 0.91): a delta increase of 5 and 15 Sharp- van der Heijde Score (SHS) points. The other outcome was time till sustained remission irrespective of DMARD therapy. Sustained remission was derived from the ESPOIR database and defined as the sustained absence of swollen joints count $(=0)$ for at least 1 year irrespective of DMARD therapy and subsequently absence of swollen joints for the entire follow-up (the maximal follow-up was 5 years). 


\section{Statistics}

Cox proportional hazards regression models using natural cubic splines were used to model the effect of the symptom duration on time to DMARD-free sustained remission. ${ }^{14}{ }^{15}$ The use of cubic spline functions allows investigation of the shape of the effect; in contrast to regular cox regression, it does not assume linearity. HRs generally have a non-linear relationship with covariates; this is not the case for log-HRs. Therefore, the log-HR for increasing symptom duration values was plotted based on the model that fitted the data best. Time-dependent receiver operator characteristic (ROC) curves were used to evaluate the discriminative capability of symptom duration; the optimal cut-off value for symptom duration was determined using Youden's index (optimal sensitivity + specificity-1). All analyses were done in the statistical software $\mathrm{R} ;{ }^{16}$ in particular, the R-packages survival and survival ROC were used. ${ }^{17} 18$

\section{RESULTS}

\section{Symptom duration in relation to log-HR on DMARD-free sustained remission}

Characteristics of the RA patients are presented in table 1. The median symptom duration at inclusion was 18.7 weeks (IQR 9.3-35.3) in the EAC and 21.3 weeks (IQR 13.4-33.5) in ESPOIR. In all, $67 \%$ and $76 \%$ of the patients started treatment with methotrexate; the majority of the remaining patients were treated with sulfasalazine or hydroxychloroquine. During 5-year follow-up, DMARD-free sustained remission was obtained in $11.5 \%(85 / 738)$ and $5.4 \%(29 / 533)$ in the EAC and ESPOIR, respectively.

First, the log-HR on DMARD-free sustained remission (the opposite of RA persistence) during 5-year follow-up was plotted against the symptom duration in the EAC (figure 1A). The log-HRs were negative, indicating a decreased chance on DMARD-free sustained remission, which equals an increased chance on disease persistence. The curve was not linear and the steepness changed at a point in time (figure 1A). For RA patients included in ESPOIR, a non-linear profile was also observed; after an almost linear start, the curve flattened towards an almost horizontal line (figure $1 \mathrm{~B}$ ). In order to comprehend the HRs more easily, the profile plotting the HR on DMARD-free sustained remission against symptom duration is presented as well (figure 1C, D).

\section{Time integrated ROC curves}

To evaluate the discriminative capability of symptom duration, time-dependent ROC curves were constructed with DMARD-free sustained remission as outcome. In both cohorts, the areas under the ROC curves were rather low: 0.61 in EAC and 0.59 in ESPOIR (figure 1E, F). These low values reduced the certainty with which the optimum of the ROC curve can be identified. The symptom duration with the best discrimination of patients with DMARD-free sustained remission from patients with persistent disease was 14.9 weeks (95\% CI 12.3 to 16.0 ) in the EAC. Similarly, the symptom duration with best combination of sensitivity and specificity was 19.1 weeks (95\% CI 12.3 to 28.0) in ESPOIR.

\section{Subanalyses on initiated DMARD}

To evaluate the influence of treatment on the relation between the log-hazard on DMARD-free sustained remission and symptom duration, analyses on 5-year follow-up data were repeated for patients treated with methotrexate and patients treated with other conventional DMARDs separately.
In both groups of patients in both cohorts, the profiles of the association between symptom duration and DMARD-free sustained remission were not linear (figure 2). The optimum symptom duration (determined using ROCs) in the EAC was 14.4 weeks (95\% CI 10.3 to 46.3 ; area under the curve (AUC) 0.61 ) for methotrexate users and 18.3 weeks (95\% CI 10.7 to 39.9; AUC 0.60) for patients treated with other DMARDs. In ESPOIR, this was 19.1 weeks (95\% CI 7.0 to 168.3 ; AUC 0.55 ) and 20.7 weeks (95\% CI 13.4 to 25.7; AUC 0.67, see online supplementary figure $\mathrm{S} 1$ ), respectively.

\section{Subanalyses on ACPA}

Because ACPA-positive and ACPA-negative RA differ in the processes underlying disease development and disease outcomes, analyses were also stratified for ACPA. In the EAC, $n=67$ (19.9\%) of ACPA-negative RA patients achieved DMARD-free sustained remission during 5 years and $n=13$ (3.5\%) of ACPA-positive RA patients. Also here, the curves plotting the log-HRs on DMARD-free sustained remission against symptom duration were not linear (data not shown). The optimum symptom duration was 11.4 weeks (95\% CI 7.7 to 79.0 ; AUC 0.56) for ACPA-positive RA patients and 15.0 weeks $(95 \% \mathrm{CI}$ 9.7 to 48.7; AUC 0.56, see online supplementary figure S2) for ACPA-negative RA patients. In ESPOIR, only $n=3(1.1 \%)$ ACPA-positive patients obtained DMARD-free sustained remission, prohibiting stratified analyses in this cohort.

\section{Subanalyses using other outcomes}

Although the outcome DMARD-free remission fits most closely to the window of opportunity hypothesis, the shape of the association between symptom duration and other outcomes was evaluated as well. First, the time till a certain level of radiographic progression was achieved (an increase of 5 and 15 SHS points from baseline onwards, respectively) was assessed. These outcomes were obtained in $294(61 \%)$ and 148 (31\%) of the RA patients after a median follow-up of 3 (IQR 1-5) and 5 (4-5) years, respectively. The cox regression analyses using these outcomes also showed non-linear curves (figure 3A, B). Sustained remission was studied as well, although this outcome might reflect an individual patient's susceptibility to certain treatments in addition to changes in underlying disease mechanisms. Sustained remission was obtained in 212 (40\%) patients. The profile was not linear here also; after an almost linear start, the steepness of the curve changed substantially at a certain point in time (figure 3C).

\section{DISCUSSION}

During the last two decades, many studies have observed that the time to intervention is associated with the efficacy of this intervention. A window of opportunity for therapeutic interventions, a time frame within which there is a disproportionate response to therapy resulting in long-term sustained benefits, is proposed to be present. The concept of this window was first hypothesised in the early 1990s. ${ }^{19}$ The studies available on this subject have either compared 'early' versus 'late' or analysed the duration as a continuous variable presuming that every increase in units of time was equally deleterious. ${ }^{1-3} 620$ Although both type of studies reported inverse associations between time to intervention and disease outcomes, the issue is still open whether there truly is an early period when RA patients respond to intervention in a fundamentally different way than they would if therapy was delayed. ${ }^{21}$ This caveat prompted us to study the shape of the association between log-HRs on achieving DMARD-free sustained remission (absence of RA 

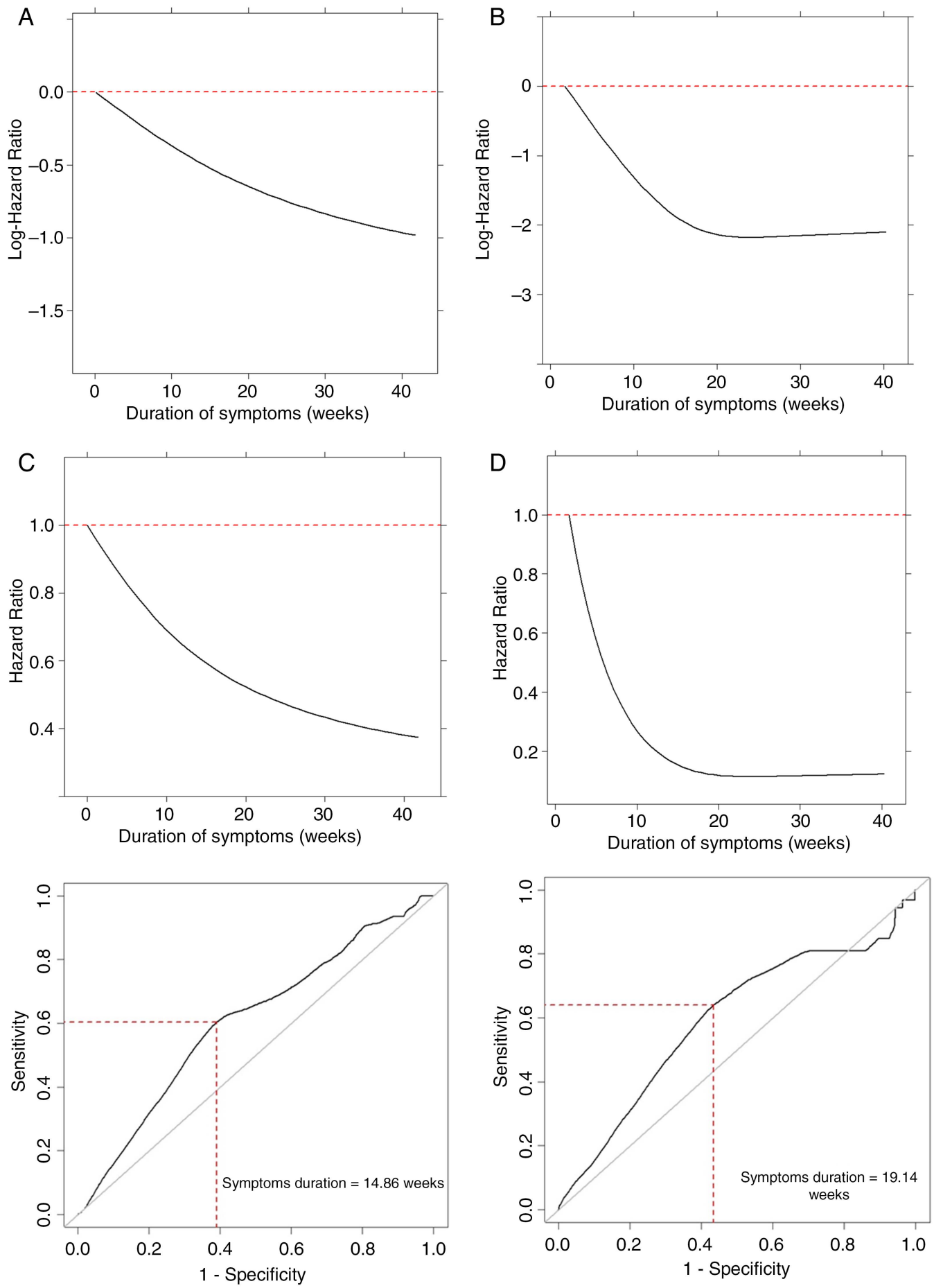

Figure 1 Symptom duration in relation to the log-hazard ( $A$ and $B)$ and HR (C and D) on DMARD-free sustained remission in rheumatoid arthritis patients in the EAC (A and C) and Evaluation et Suivi de POlyarthrites Indifférenciées Récentes (ESPOIR) (B and D) and the HR in the EAC (C) and ESPOIR (D) during 5-year follow-up. Time-dependent receiver operator characteristic curves of symptom duration with DMARD-free sustained remission as outcome in the EAC (E) and ESPOIR (F). (E and F) The symptom duration with the best discriminative ability was determined using Youden's index. DMARD, disease modifying antirheumatic drug; EAC, Early Arthritis Clinic.

persistence) and symptom duration in RA patients treated with conventional DMARDs. The patients studied were included in ESPOIR and the Leiden EAC; previous studies on these cohorts have already shown that a prolonged symptom association was statistically significantly associated with an unfavourable course of RA. ${ }^{2}$ We here applied methodology that allowed elucidating the shape of the relationship. We observed a non-linear association; the log-hazard on DMARD-free sustained remission decreased after a certain symptom duration. These data suggest that a confined period in which the disease is more susceptible to treatment is present indeed.

The duration of the window is unknown. In 1991, the window was supposed to endure 2 years ${ }^{22}$ and at present it is considered to be the first 3 months. However, these suggestions are based on expert opinion rather than on scientific data. Although the cut-off of 3 months or 12 weeks is often referred to, ${ }^{82}{ }^{24}$ we did not find literature with data supporting this time-point. Several studies compared patients with symptoms for $<$ or $\geq 3$ 

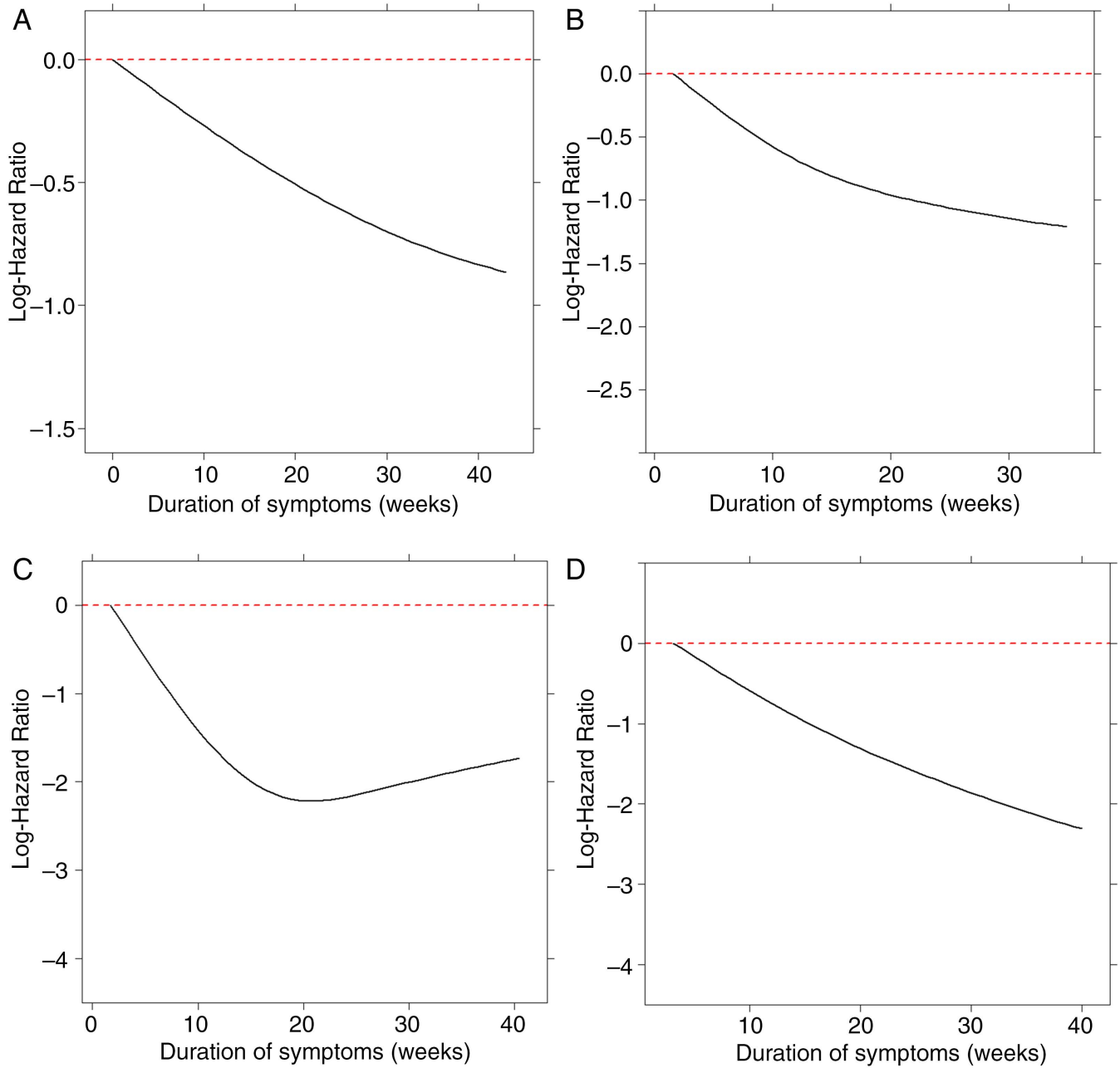

Figure 2 Symptom duration in relation to the log-hazard on DMARD-free sustained remission during 5-year follow-up in rheumatoid arthritis patients treated with methotrexate (A and C) or other conventional DMARDS (B, D) in the Leiden EAC (A and B) and Evaluation et Suivi de POlyarthrites Indifférenciées Récentes (ESPOIR) (C and D). DMARD, disease modifying antirheumatic drug; EAC, Early Arthritis Clinic.

months, ${ }^{2} 67$ but a rationale for this categorisation was not provided and it is unknown whether results would have been even stronger when another cut-off was chosen. In the present data, the steepness of the curve plotting the log-HR against symptom duration diminished around 15-20 weeks after symptom onset (figure 1). It cannot be concluded that the window 'is closed' after this period, but the data of the present cohorts clearly showed that the hazard on remission was less after this period, and so possibly it 'starts to close' at this point in time. In other words, we do not suggest that DMARD treatment after a certain window is futile, but that initiating a DMARD in this particular window might yield a better outcome. In case a patient is identified after this period has passed, DMARD therapy should certainly not be withheld.

The symptom duration with the optimum discriminative ability of disease persistence was estimated at 14.9 weeks in the EAC and 19.1 weeks in ESPOIR. The time point at which the curve plotting the log-HR against symptom duration started to flatten does not necessarily coincide with the symptom duration with the best Youden's index determined using an ROC. Nonetheless, in the present data both time indications were roughly similar. Importantly, the time estimation obtained using the ROC curves should be interpreted with care. First, because the ROC curves were relatively flat and the AUCs low, making the identification of a single time point less reliable compared with ROC curves with high AUCs. This uncertainty is reflected by the broad CIs. Second, the exact dates at which patients started to take DMARDs were not known in all patients (in the EAC, these data were incomplete; in ESPOIR, the median duration between inclusion and DMARD start was 1.4 weeks). We therefore performed the analyses defining symptom duration as the period between symptom onset and the first visit. Consequently, the obtained time estimation may be too 'narrow'. Third, the time indications are obtained on group level and may not be identical in every individual patient with RA. Because of these limitations, we cannot give definite answers on the length of the window of opportunity. Still, the observed results on symptom duration are rather consistent, which suggests some validity of these results.

The validity of the results on non-linear associations of symptom duration with the course of RA are strengthened by the finding that non-linear curves were also observed for radiographic progression and sustained remission. The curves on DMARD-free sustained remission of the EAC and ESPOIR cohort were not completely similar as the change in steepness was more profound in the results of the ESPOIR cohort. Nevertheless, when evaluating the results of both cohorts and the different outcomes, the general picture is that the association of symptom duration with the chance of either a favourable outcome (remission) or unfavourable outcome (joint damage) is not linear. 

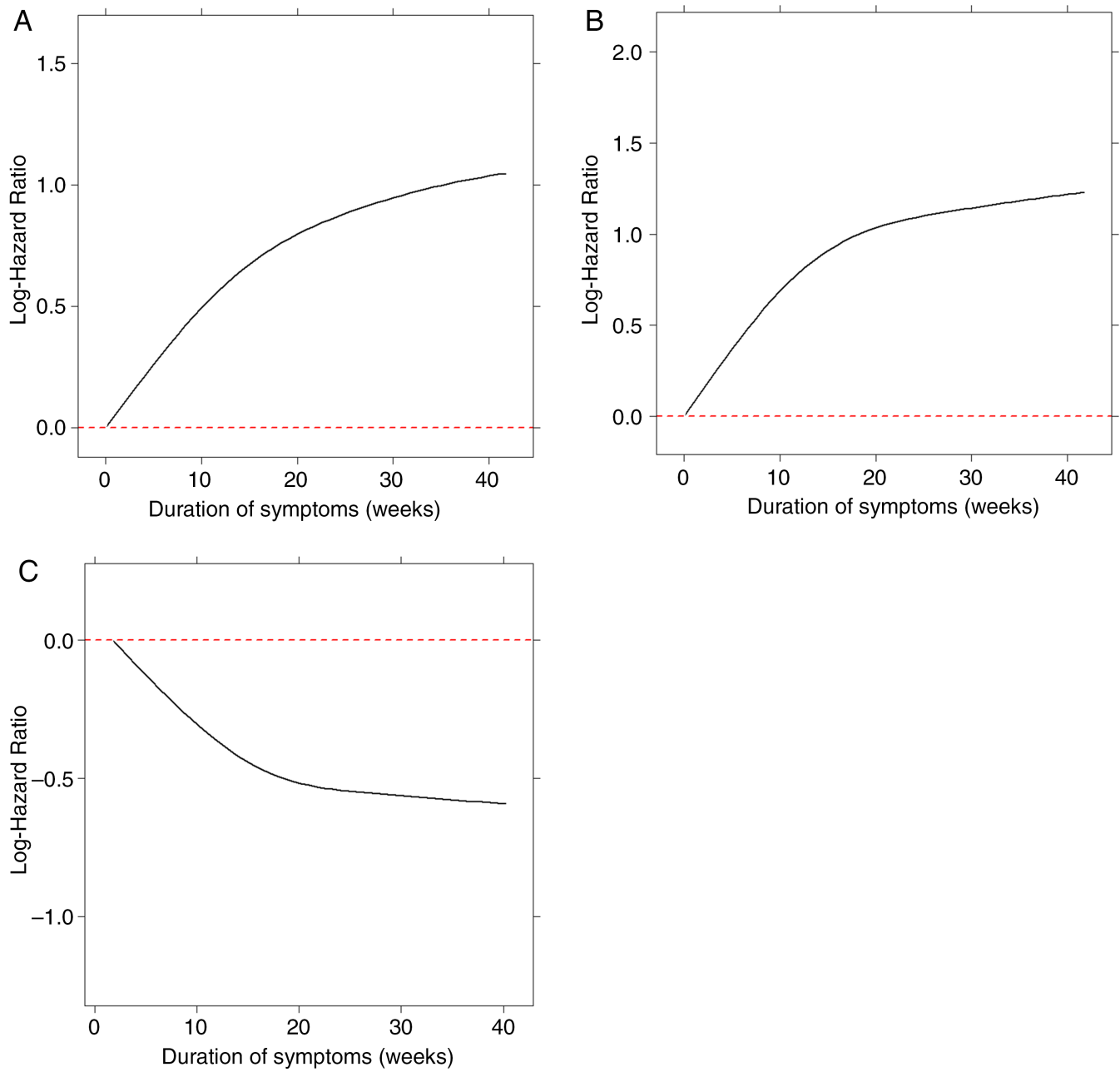

Figure 3 Symptom duration in relation to the log-hazard on an increase in SHS scores of 5 (A) and 15 (B) from inclusion onwards in the Leiden EAC and the log-hazard on sustained remission irrespective of DMARD therapy (C) in Evaluation et Suivi de POlyarthrites Indifférenciées Récentes (ESPOIR), all assessed during 5-year follow-up in rheumatoid arthritis patients. Achievement of an increase of SHS of 5 and 15 points is an unfavourable outcome and therefore the log-HRs were above 0 . Sustained remission, in contrast, is a favourable outcome and therefore the HRs were below 0. DMARD, disease modifying antirheumatic drug; EAC, Early Arthritis Clinic.

This study has limitations. It has been noted previously that studies evaluating the symptom or disease duration should indicate precisely how symptom onset or disease onset is defined in order to allow comparisons between studies. ${ }^{13}$ In both cohorts, the onset of symptoms was defined identically as the first symptoms noticed by patients themselves. The fact that the symptom start was reported by patients may have induced some heterogeneity due to inter-individual differences in symptom awareness or symptom recollection. ${ }^{25}$ Another issue is that we evaluated data of longitudinal observational cohort studies. These data reflect the daily care of patients and decisions to start and stop DMARDs were left to the patients and rheumatologists and not protocolised. In ESPOIR, mainly in the first years of its existence, quitting DMARD therapy was uncommon. Consequently, the observed frequency of DMARD-free sustained remission may be underestimated. Finally, whether DMARD-free sustained remission was achieved was determined slightly differently in the cohorts. In the EAC, all medical files were checked to ensure that DMARD-free sustained remission was present. In ESPOIR, the data of the structured visits with yearly intervals were studied. It is possible that more patients included in ESPOIR would have achieved DMARD-free sustained remission when all information present in medical files was evaluated. Though, differences in common practice on discontinuing DMARD therapy might be the most important cause for the higher frequency of DMARD-free sustained remission in the EAC than in ESPOIR.

It is not known which DMARD is most effective in taking advantage of the early, treatment susceptible, disease period. None of the patients studied were treated with biologics as first therapy. Since methotrexate is the treatment of first choice according to European league against rheumatism (EULAR) recommendations, ${ }^{26}$ patients treated with methotrexate were studied in detail. The results of this subanalysis were in line with the results of the total group. Although it is known that methotrexate therapy reduces the severity of radiographic progression, ${ }^{27} 28$ thus far evidence demonstrating that methotrexate is able to increase the chance on DMARD-free sustained remission when initiated early is absent. Current data therefore add importantly to the conception of methotrexate being disease modifying. ${ }^{26}$

The biological mechanisms underlying the concept of the window of opportunity are unclear. Pathophysiologically, ACPA-positive and ACPA-negative RA are considered to be different subsets of RA. Analyses stratified for ACPA were possible in one cohort and revealed a slightly shorter window in ACPApositive than in ACPA-negative patients (11.4 vs 15.0 weeks). Because the CIs were broad, no definite conclusions can be drawn 
on these comparisons, though the tendency in the data may lead to the speculation that the presence of ACPA is related to a master-switch leading to an earlier closing of the window.

Present guidelines on the treatment of RA contain indications on the time ideally elapsed until access to rheumatological care is obtained or treatment is started. ${ }^{26} 29$ More studies are required to determine what is 'in time' or 'too late' for specific groups of RA patients.

In conclusion, the present study demonstrated that the HRs on DMARD-free sustained remission decreased considerably before a certain time point. This study is the first providing strongly suggestive evidence that a confined period in which RA is more susceptible to treatment exits. Further proof might be obtained by performing clinical trials in patients with symptoms of very recent onset randomising for direct or delayed treatment. However, given the present knowledge this may be considered unethical.

Acknowledgements We would like to acknowledge Stefaan Bral for his work in checking medical files of EAC patients on DMARD use during his internship at the rheumatology department in the LUMC. For ESPOIR, we wish to thank Nathalie Rincheval who did expert monitoring and data management, G Tobon who did $X$-ray reading, S Martin (Paris Bichat) who did all the central dosages of CRP, IgA and IgM rheumatoid factor and anti-CCP antibodies, and all the investigators who recruited and followed the patients ( $F$ Berenbaum, Paris-Saint Antoine, MC. Boissier, Paris-Bobigny; A Cantagrel, Toulouse; B Combe, Montpellier, M Dougados, Paris-Cochin; P Fardellone and P Boumier Amiens; B Fautrel, P Bourgeois, Paris-La Pitié, RM Flipo, Lille; P Goupille, Tours; F Liote, Paris-Lariboisière; X Le Loet, Rouen; X Mariette, Paris Bicêtre; O Meyer, Paris Bichat; A Saraux, Brest; T Schaeverbeke, Bordeaux; J Sibilia, Strasbourg).

Contributors JABvN and RT analysed the data. AHMvdH-vM and JABvN contributed to the conception, design and interpretation of the data. AHMvdH-vM drafted the article. JABvN, RT, CG-V, and BF revised the article for important intellectual content. All authors gave final approval for the version to be published.

Funding The research leading to these results was funded by a Vidi-grant of the Netherlands Organisation for Scientific Research (to AHMvdH-vM), a grant of the Dutch Arthritis Foundation (Reumafonds) and by a grant of the EuroTEAM consortium. An unrestricted grant from Merck Sharp and Dohme (MSD) was allocated for the first 5 years of the ESPOIR cohort study. Two additional grants from INSERM were obtained to support part of the biological database of ESPOIR. The French Society of Rheumatology, Abbott and Wyeth-Pfizer also supported the ESPOIR cohort study.

Competing interests None.

Ethics approval Local ethics committees.

Provenance and peer review Not commissioned; externally peer reviewed.

\section{REFERENCES}

1 van Nies JA, Krabben A, Schoones JW, et al. What is the evidence for the presence of a therapeutic window of opportunity in rheumatoid arthritis? A systematic literature review. Ann Rheum Dis 2014:73:861-70.

2 van der Linden MP, le Cessie S, Raza K, et al. Long-term impact of delay in assessment of patients with early arthritis. Arthritis Rheum 2010;62:3537-46.

3 Anderson JJ, Wells $\mathrm{G}$, Verhoeven $\mathrm{AC}$, et al. Factors predicting response to treatment in rheumatoid arthritis: the importance of disease duration. Arthritis Rheum 2000;43:22-9

4 Feldman DE, Bernatsky $S$, Houde $M$, et al. Early consultation with a rheumatologist for RA: does it reduce subsequent use of orthopaedic surgery? Rheumatology (Oxford) 2013;52:452-9.

5 Symmons DP, Jones MA, Scott DL, et al. Longterm mortality outcome in patients with rheumatoid arthritis: early presenters continue to do well. J Rheumatol 1998:25:1072-7.
6 Nell VP, Machold KP, Eberl G, et al. Benefit of very early referral and very early therapy with disease-modifying anti-rheumatic drugs in patients with early rheumatoid arthritis. Rheumatology (Oxford) 2004;43:906-14.

7 Lukas C, Combe B, Ravaud P, et al. Favorable effect of very early disease-modifying antirheumatic drug treatment on radiographic progression in early inflammatory arthritis: Data from the Etude et Suivi des polyarthrites indifferenciees recentes (study and followup of early undifferentiated polyarthritis). Arthritis Rheum 2011;63:1804-11.

8 Raza K. The Michael Mason prize: early rheumatoid arthritis--the window narrows. Rheumatology (Oxford) 2010;49:406-10.

9 Egsmose C, Lund B, Borg G, et al. Patients with rheumatoid arthritis benefit from early 2 nd line therapy: 5 year followup of a prospective double blind placebo controlled study. J Rheumatol 1995;22:2208-13.

10 Tsakonas E, Fitzgerald AA, Fitzcharles MA, et al. Consequences of delayed therapy with second-line agents in rheumatoid arthritis: a 3 year followup on the hydroxychloroquine in early rheumatoid arthritis (HERA) study. J Rheumatol 2000;27:623-9.

11 de Rooy DP, van der Linden MP, Knevel R, et al. Predicting arthritis outcomes--what can be learned from the Leiden Early Arthritis Clinic? Rheumatology (Oxford) 2011;50:93-100

12 Combe B, Benessiano J, Berenbaum F, et al. The ESPOIR cohort: a ten-year follow-up of early arthritis in France: methodology and baseline characteristics of the 813 included patients. Joint Bone Spine 2007;74:440-5.

13 Raza K, Saber TP, Kvien TK, et al. Timing the therapeutic window of opportunity in early rheumatoid arthritis: proposal for definitions of disease duration in clinical trials. Ann Rheum Dis 2012;71:1921-3.

14 Heinzl H, Kaider A, Zlabinger G. Assessing interactions of binary time-dependent covariates with time in cox proportional hazards regression models using cubic spline functions. Stat Med 1996;15:2589-601.

15 Heinzl H, Kaider A. Gaining more flexibility in Cox proportional hazards regression models with cubic spline functions. Comput Methods Programs Biomed 1997:54:201-8.

16 R Foundation for Statistical Computing VA. R Core Team (2013). R: A language and environment for statistical computing. 2014. Ref Type: Online Source

17 Therneau TM, Grambsch PM. Modeling Survival Data: Extending the Cox Model. New York: Springer, 2000

18 Heagerty PJ, Lumley T, Pepe MS. Time-dependent ROC curves for censored survival data and a diagnostic marker. Biometrics 2000;56:337-44.

19 Emery P. The Dunlop-Dottridge Lecture: prognosis in inflammatory arthritis: the value of HLA genotyping and the oncological analogy. J Rheumatol 1997;24:1436-42.

20 Green M, Marzo-Ortega H, McGonagle D, et al. Persistence of mild, early inflammatory arthritis: the importance of disease duration, rheumatoid factor, and the shared epitope. Arthritis Rheum 1999:42:2184-8.

21 O'Dell JR. Treating rheumatoid arthritis early: a window of opportunity? Arthritis Rheum 2002;46:283-5.

22 Wolfe F, Hawley DJ, Cathey MA. Clinical and health status measures over time: prognosis and outcome assessment in rheumatoid arthritis. J Rheumatol 1991;18:1290-7.

23 Quinn MA, Emery P. Window of opportunity in early rheumatoid arthritis: possibility of altering the disease process with early intervention. Clin Exp Rheumatol 2003;21 (5 Suppl 31):S154-7.

24 Boers M. Understanding the window of opportunity concept in early rheumatoid arthritis. Arthritis Rheum 2003:48:1771-4.

25 Amjadi S, Khanna D, Park GS, et al. Dating the "window of therapeutic opportunity" in early rheumatoid arthritis: accuracy of patient recall of arthritis symptom onset. J Rheumatol 2004;31:1686-92.

26 Smolen JS, Landewe R, Breedveld FC, et al. EULAR recommendations for the management of rheumatoid arthritis with synthetic and biological disease-modifying antirheumatic drugs: 2013 update. Ann Rheum Dis 2014;73:492-509.

27 Landewe RB, Boers M, Verhoeven AC, et al. COBRA combination therapy in patients with early rheumatoid arthritis: long-term structural benefits of a brief intervention. Arthritis Rheum 2002;46:347-56.

28 Mottonen $\mathrm{T}$, Hannonen $\mathrm{P}$, Korpela $\mathrm{M}$, et al. Delay to institution of therapy and induction of remission using single-drug or combination-disease-modifying antirheumatic drug therapy in early rheumatoid arthritis. Arthritis Rheum 2002;46:894-8.

29 Luqmani R, Hennell S, Estrach C, et al. British Society for Rheumatology and british health professionals in Rheumatology guideline for the management of rheumatoid arthritis (the first two years). Rheumatology (Oxford) 2006;45:1167-9. 